\title{
RADIOLOGICAL PATTERN OF PULMONARY TUBERCULOSIS IN DIABETICS VISITING A TERTIARY CARE HOSPITAL
}

\author{
Sindhura Goganti ${ }^{1}$, S. Lithya Kumari², Ashly Thomas ${ }^{3}$, B. Hari Prasad ${ }^{4}$, B. Rajagopalan 5 , C. Chandrasekar 6 \\ ${ }^{1}$ Assistant Professor, Department of Pulmonary Medicine, Sri Ramachandra Medical College and Research Institute, Sri Ramachandra \\ University, Porur, Chennai. \\ ${ }^{2}$ Postgraduate Student, Department of Pulmonary Medicine, Sri Ramachandra Medical College and Research Institute, Sri \\ Ramachandra University, Porur, Chennai. \\ ${ }^{3}$ Allied Health Science, Department of Pulmonary Medicine, Sri Ramachandra Medical College and Research Institute, Sri \\ Ramachandra University, Porur, Chennai. \\ ${ }^{4}$ Professor, Department of Pulmonary Medicine, Sri Ramachandra Medical College and Research Institute, Sri Ramachandra University, \\ Porur, Chennai. \\ 5Professor, Department of Pulmonary Medicine, Sri Ramachandra Medical College and Research Institute, Sri Ramachandra University, \\ Porur, Chennai. \\ ${ }^{6}$ Professor and Head, Department of Pulmonary Medicine, Sri Ramachandra Medical College and Research Institute, Sri Ramachandra \\ University, Porur, Chennai.
}

\begin{tabular}{l}
\hline ABSTRACT \\
BACKGROUND \\
Tuberculosis (TB) is an infectious disease caused by Mycobacterium tuberculosis (MTB). Pulmonary TB is a major public health \\
problem in developing countries especially in India. India is the country with the highest burden of TB. The number of people with \\
diabetes has risen from 108 million in 1980 to 422 million in 2014. Diabetes is fuelling the spread of Tuberculosis. In India, in \\
2000 , calculation suggested that diabetes accounted for $14.8 \%$ of PTB and $20.2 \%$ of Smear-Positive TB patients.
\end{tabular}

\section{MATERIALS AND METHODS}

A descriptive study was conducted in the Department of Chest \& TB in Sri Ramachandra Medical Centre.

\section{RESULTS}

A total of 123 patients were taken and lower zone distribution was found to be maximum among them.

\section{CONCLUSION}

It is observed from the study that sputum positivity is more than sputum negativity in Diabetics. It is also found that cavity was $40 \%$ in diabetics compared to general population.

\section{KEYWORDS}

Diabetes Mellitus, Tuberculosis, Sputum Positivity, Lower Zone.

HOW TO CITE THIS ARTICLE: Goganti S, Kumari SL, Thomas A, et al. Radiological pattern of pulmonary tuberculosis in diabetics visiting a tertiary care hospital. J. Evolution Med. Dent. Sci. 2017;6(85):5920-5923, DOI: 10.14260/jemds/2017/1287

\section{BACKGROUND}

Tuberculosis (TB) and diabetes mellitus (DM) are both important health issues affecting our society. The link of Diabetes Mellitus and Tuberculosis is more prominent in developing countries where Tuberculosis is endemic and the burden of diabetes mellitus is increasing having a negative impact on Indian population. The association between diabetes and tuberculosis may be the next challenge for control of global tuberculosis worldwide.

In recent decades, with the increasing prevalence of $\mathrm{TB}$, particularly Multidrug Resistant TB (MDR-TB), and DM cases in the world, the relationship is re-emerging as a significant public health problem. Proper planning and management are necessary to reduce the dual burden of diabetes mellitus and Tuberculosis.

'Financial or Other Competing Interest': None.

Submission 08-08-2017, Peer Review 08-10-2017,

Acceptance 14-10-2017, Published 23-10-2017.

Corresponding Author:

Dr. S. Lithya Kumari,

\#156 A, MTH Road,

Villivakkam, Chennai-49.

E-mail: lithyasam23@gmail.com

DOI: $10.14260 /$ jemds $/ 2017 / 1287$

\section{(c) (i) $(9)$}

J. Evolution Med. Dent. Sci./eISSN- 2278-4802, pISSN- 2278-4748/ Vol. 6/ Issue 85/ Oct. 23, 2017
Diabetes prevalence has increased worldwide as a result of population ageing, urbanisation, changes in diet and reduced physical activity patterns resulting in increasing obesity. About $80 \%$ of the 415 million estimated DM cases globally are from low and middle-income countries and the DM prevalence is projected to rise most steeply in regions with high TB incidence over the next 30 years. A systematic review of 13 observational studies found that DM increases the risk of TB by three-fold (relative risk 3.11; 95\% CI 2.274.26). This illustrates the complexity of studying DM as a risk factor for TB given the heterogeneity in DM populations worldwide with respect to their age, access to healthcare, level of glucose control, and the type and number of DM complications and medications. Furthermore, co-occurrence of DM with other host characteristics can further synergise TB risk among DM patients, as suggested for DM plus smoking, micro and macrovascular complications of DM, and even their social environment. This emphasises the need for studies reporting a thorough characterisation of DM and other host factors with multivariable analysis in order to reach reliable conclusions. ${ }^{[1]}$

The first report of the association between DM and TB was documented by Avicenna (980-1027 AD) over one thousand years ago. Since that time, the relationship between 
diabetes mellitus (DM) and tuberculosis (TB), and the nature of their interaction with regards to comorbidity are largely suggested by numerous epidemiological studies. In the early $20^{\text {th }}$ century, the effect of DM on TB was a large concern of investigators, but this was somewhat neglected in the second half of the $20^{\text {th }}$ century.[2,3]

Pulmonary TB is a major public health problem in developing countries especially in India. India is a country with the highest burden of TB. In India, 1.9 Million are diagnosed with TB every year. The World Health Organization (WHO) statistics for 2015 give an estimated incidence of 2.2 million cases of TB for India out of a global incidence of 9.6 million.

The number of people with diabetes has risen from 108 million in 1980 to 422 million in 2014.[4].The global prevalence of diabetes among adults (over 18 years of age) has risen from $4.7 \%$ in 1980 to $8.5 \%$ in 2014.[5] Almost half of all deaths attributable to high blood glucose occur before the age of 70 years. WHO projects that diabetes will be the $7^{\text {th }}$ leading cause of death in 2030 .

One in three people around the world (two billion people) holds a latent Tuberculosis infection somewhere in their body, where it can remain dormant through one's whole life. People with a weak immune system as a result of chronic diseases such as diabetes, are at a higher risk of progressing from latent to active TB. Currently, Diabetes is fuelling the spread of Tuberculosis. In India, in 2000, calculation suggested that diabetes accounted for $14.8 \%$ of PTB and $20.2 \%$ of Smear-Positive TB patients.[6]

\section{Pathophysiology}

Tuberculosis (TB) is an infectious disease caused by the bacterium Mycobacterium tuberculosis (MTB).[7] Though it primarily affects the lungs, tuberculosis can also affect other parts of the body such as intestines, meninges, bones and joints, lymph glands, skin and other tissues. Pulmonary TB is generally classified into Primary, Post-primary TB, Progressive Primary TB, each having distinct Radiological pattern.

Although the definite pathophysiological mechanism of the effect of Diabetes mellitus as a predisposing risk factor for TB is unknown, some hypotheses are suggested: depressed cellular immunity, dysfunction of alveolar macrophages, low levels of interferon gamma, pulmonary microangiopathy, and micronutrient deficiency.[8,9]

The pathophysiology of tuberculosis is complex. Acquisition of the infection is primarily dependent on exogenous factors; however, reactivation of disease is largely under the influence of immune sufficiency. In spite of frequent studies about the link between DM and active tuberculosis, the effect of DM on the frequency of latent TB has been less investigated. The few existing reports about a higher prevalence of latent TB infection among diabetics have been confounded by an absence of control groups. Results of one study showed the reaction to purified protein derivate (PPD) is significantly correlated to the degree of hyperglycaemia. Many studies suggest that DM is associated with the clinical presentation of TB. Namely, TB-DM patients (versus TB-no DM) are more likely to present with pulmonary (versus extra-pulmonary), cavitary (versus noncavitary) and sputum smear-positive TB at diagnosis. During the course of TB treatment, TB-DM patients take longer to convert from sputum smear-positive to negative.[10]
Pulmonary TB accounts for $70-80 \%$ of the cases, and it is generally accepted that immune compromise facilitates haematogenous dissemination of $\mathrm{Mtb}$, predisposing to extrapulmonary TB. Such is the case of TB patients with HIVAIDS or those taking TNF blockers. This contrasts with TBDM patients who are less likely to present with extrapulmonary TB. This may be due to a hyper-reactive cellmediated immune response to Mtb in DM patients that may be suboptimal for containing Mtb growth within the lung, but effective for preventing its dissemination and reactivation. ${ }^{[10]}$

\section{Synergy of Tuberculosis and Diabetes Mellitus}

If diabetes can predispose a patient to tuberculosis, can infection with tuberculosis lead to diabetes mellitus? Infections, including tuberculosis, often worsen glycaemic control in diabetic patients, and poorly controlled diabetes might in turn augment the severity of infections. Some studies suggest that tuberculosis can even cause diabetes in those not previously known to be diabetic. Many studies have used oral glucose tolerance testing to show that patients with tuberculosis have higher rates of glucose intolerance than community controls. Whereas the high incidence of abnormal oral glucose tolerance found in tuberculosis patients is of concern, it is unclear whether glucose intolerance or diabetes mellitus was truly an incident, or whether prevalent diabetes mellitus was being newly diagnosed in patients receiving expanded medical services related to tuberculosis treatment. Also, the implications of these findings depend on whether diabetes mellitus persists in these patients, and whether its presence is substantially more common with tuberculosis than with other infectious diseases.

In a study in Nigeria, tuberculosis patients with impaired glucose tolerance had normal tests after 3 months of tuberculosis treatment. In Turkey, oral glucose tolerance tests were given to 58 patients with active tuberculosis and 23 patients with community-acquired pneumonia. Of those with tuberculosis, $10 \%$ were glucose intolerant and $9 \%$ had diabetes; of patients with community-acquired pneumonia, none had glucose intolerance and $17 \%$ were diabetic. All patients had normal tests, 3 months and 2 years after the start of treatment. The latter two studies suggest that infection causes reversible glucose intolerance and that this effect is not specific to tuberculosis. In Indonesia, 13\% (60 of 454) of patients with tuberculosis had diabetes, compared with $3 \cdot 2 \%$ (18 of 556 ) of age-matched and sex-matched controls from the same residential unit; for $60 \%$ of these patients, diabetes was a new diagnosis. Whereas impairment of glucose metabolism probably preceded tuberculosis in these patients rather than the reverse, these data underscore the importance of screening tuberculosis patients for diabetes.[10]

\section{Aims of the Study}

- To identify the various radiological patterns of pulmonary tuberculosis in the diabetic adults.

- To find out the pattern of distribution of tuberculosis and its incidence based on the lung fields in diabetic patients.

\section{MATERIALS AND METHODS}

A descriptive study was conducted in the Department of Chest \& TB in Sri Ramachandra Medical Centre. Diabetic 
patients between 40-70 years of age who had symptoms suggestive of pulmonary tuberculosis were included in the study. Both sputum positive and negative patients were included. Each of them were then subjected to radiological investigations, that is chest X-rays (PA view) were taken. Diabetics were based on WHO criteria.

\section{RESULTS}

In this study, among 123 diabetic patients in the age range 40 to 70 years, 97 of them were male and 26 were female. Maximum age was 70 years and minimum age was 40 years. The mean age was $55+5$ years. Out of 123,100 patients were sputum positive, 23 were sputum negative.

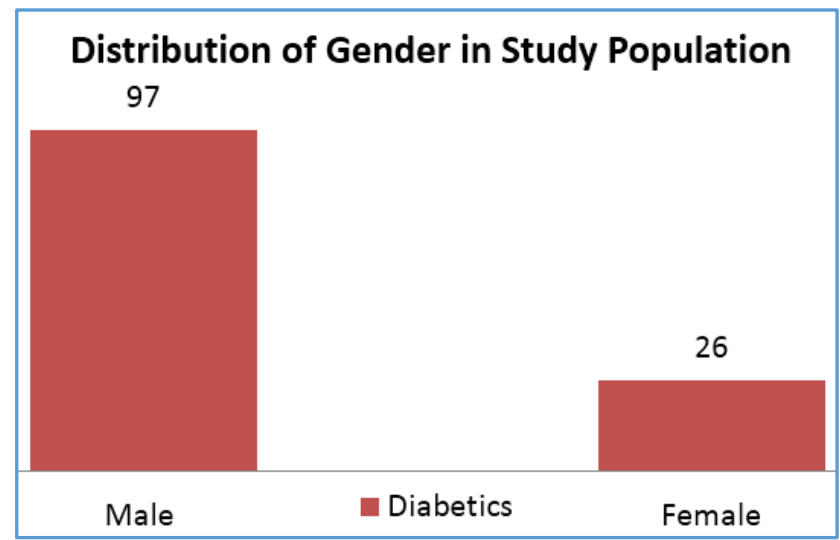

Table 1. Gender Distribution

\begin{tabular}{|c|c|c|}
\hline Zone & Population & Distribution \% \\
\hline Right upper zone & 11 & $8.9 \%$ \\
\hline Right middle zone & 18 & $14.6 \%$ \\
\hline Right lower zone & 30 & $24.3 \%$ \\
\hline Left upper zone & 3 & $2.5 \%$ \\
\hline Left middle zone & 24 & $19.5 \%$ \\
\hline Left lower zone & 37 & $30 \%$ \\
\hline \multicolumn{2}{|c|}{ Table 2. Distribution of Cases } \\
According to Zone of Lung Involvement
\end{tabular}

Normally, chest x- ray shows lesions in upper lobe, but in diabetics in our study, it showed that lower zone distribution of tuberculosis was more common than upper zone distribution of tuberculosis. Almost $54.3 \%$ of the cases that is 67 cases out of the total 124 had lower zone distribution of tuberculosis. Following this was the middle zone distribution of tuberculosis. The least affected was the upper zone.

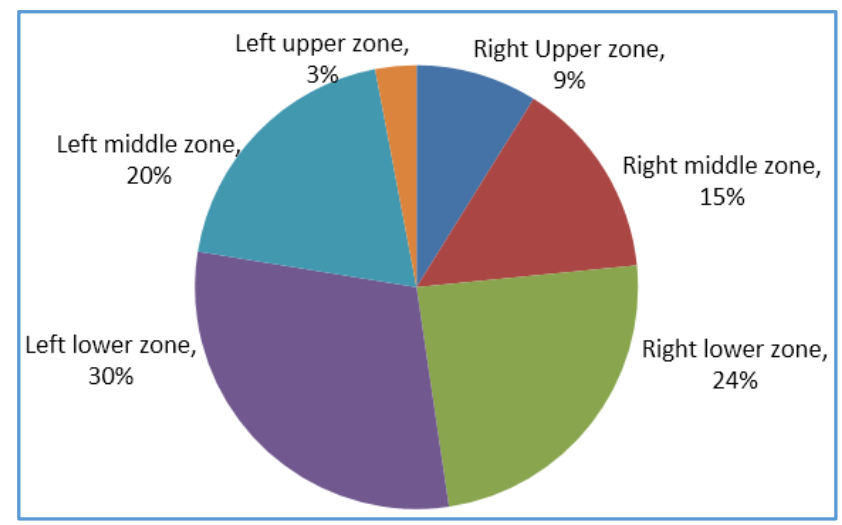

Table 3. Pie Chart of Lung Field Distribution

\begin{tabular}{|c|c|c|}
\hline X-ray Findings & $\begin{array}{c}\text { Number of } \\
\text { Patients }\end{array}$ & Percentage \\
\hline Multilobar involvement & 27 & $21.9 \%$ \\
\hline Upper zone involvement & 14 & $11.3 \%$ \\
\hline Middle zone involvement & 42 & $34.0 \%$ \\
\hline Lower zone involvement & 67 & $54.3 \%$ \\
\hline Cavitary lesions & 50 & $40.5 \%$ \\
\hline Pleural effusion & 3 & $2.4 \%$ \\
\hline
\end{tabular}

Table 4. Frequency of Different Chest $X$-rays Findings

Among the various patterns of radiological findings, the lower zone infiltrates were more common as shown above in the table, among the diabetics.

\section{DISCUSSION}

Historically, the incidence of tuberculosis in patients with diabetes has been high.[11][12]A prospective cohort study showed that patients with DM and TB have more severe clinical manifestations, delayed sputum conversion and a higher probability of treatment failure, recurrence and relapse. It has been reported that patients with TB and diabetes are older, more likely to have haemoptysis, pulmonary cavitations, be smear positive at diagnosis, and remain positive at the end of the first or second month of treatment.[13]

In our study also, we found that out of 123,100 patients were sputum positive, 23 were sputum negative.

The radiographic presentation of tuberculosis depends on many factors, including duration of illness and host immune status. In 1927, Sosman and Steidl[14] reported that a large proportion of diabetic patients with tuberculosis had lowerlung involvement, whereas non-diabetic patients usually had upper-lobe infiltrates. Subsequent studies in the 1970s and 1980 s corroborated this finding, $[15,16]$ and it was widely believed that pulmonary tuberculosis in diabetic patients presented with an atypical radiographic pattern and distribution, particularly lower-lung involvement.

There have been conflicting findings regarding the effect of DM on the radiologic characteristics of pulmonary tuberculosis. Concerning the distribution of pulmonary involvement, some studies did not find any difference between DM and non-DM cases. However, there were other studies that showed a higher incidence of lower lobe involvement among DM-TB cases. Also, there was no significant difference in the frequency of pleural effusions or isolated pleural TB between patients with and without DM.

Although some reported the opposite it seems that cavitary lesions are more common among diabetic patients especially cavitary nodular lesions. Some have suggested this difference may be apparent among uncontrolled DM cases. Another factor related to the frequency of cavitary lesions was insulin dependency. Furthermore, one study showed an association between lower lung field involvement and female gender or age greater than 40 years. Also, it has been suggested that severe pulmonary involvement in DM patients may be associated with smoking.[10]

Clinically, this is important because lower-lobe tuberculosis might be misdiagnosed as community-acquired pneumonia or cancer. Also, patients with pulmonary tuberculosis that do not have upper-lobe involvement are less likely to have positive sputum smears and cultures.[17] 
Whereas in one series, $20 \%$ of patients with diabetes mellitus presented with lower-lobe involvement,[15] in other studies, lower-lobe involvement was only seen in $1 \cdot 8 \%$ (8 of 438 patients) and $8 \cdot 3 \%$ ( 1 of 12 patients). ${ }^{[18,19]}$ Furthermore, patients with pulmonary tuberculosis that do not have upper lobe involvement are less likely to have positive sputum smears and cultures.[20]

Similar to other studies as shown above, in our study also we found lower zone involvement more than other zones especially among the diabetics. Almost $54.3 \%$ of the cases that is 67 cases out of the total 124 had lower zone distribution of tuberculosis.

TB-DM cases are more likely to remain sputum smearpositive after completion of the intensive phase of treatment, and this outcome is an early predictor of treatment failure (sputum smear or culture positivity at five months or later during treatment), which is also more likely in TB-DM versus TB-no DM and TB-DM patients should be managed differently from TB-no DM.

\section{CONCLUSION}

It is observed from the study that sputum positivity is more than sputum negativity in Diabetics. Lower zone distribution of infiltrates is more in comparison to other zones. There is a male preponderance probably due to increased exposure or due to under reporting among the women.

\section{REFERENCES}

[1] Restrepo BI. Diabetes and tuberculosis. Microbiol Spectr. 2016;4(6). doi: 10.1128/microbiolspec.TNMI7 0023-2016.

[2] Chang JT, Dou HY, Yen CL, et al. Effect of type 2 diabetes mellitus on the clinical severity and treatment outcome in patients with pulmonary tuberculosis: a potential role in the emergence of multidrug-resistance. J Formos Med Assoc 2011;110(6):372-81.

[3] Restrepo BI. Convergence of the tuberculosis and diabetes epidemics: renewal of old acquaintances. Clin Infect Dis 2007;45(4):436-8.

[4] Mathers CD, Loncar D. Projections of global mortality and burden of disease from 2002 to 2030. PLoS Med 2006;3(11):e442.

[5] Global report on diabetes. World Health Organization, Geneva, 2016.

[6] Stevenson CR, Forouhi NG, Roglic G, et al. Diabetes and tuberculosis: the impact of the diabetes epidemic on tuberculosis incidence. BMC Public Health 2007;7:234.
[7] Tuberculosis fact sheet. WHO, 2015.

[8] Webb EA, Hesseling AC, Schaaf HS, et al. High prevalence of mycobacterium tuberculosis infection and disease in children and adolescents with type 1 diabetes mellitus. Int $\mathrm{J}$ Tuberc Lung Dis 2009;13(7):868-74.

[9] Ottmani SE, Murray MB, Jeon CY, et al. Consultation meeting on tuberculosis and diabetes mellitus: meeting summary and recommendations. Int J Tuberc Lung Dis 2010;14(12):1513-7.

[10] Baghaei P, Marjani M, Javanmard P, et al. Diabetes mellitus and tuberculosis facts and controversies. Journal of Diabetes \& Metabolic Disorders 2013;12(1):58.

[11] Barach J. Historical facts in diabetes mellitus. Ann Med Hist 1928;10:387.

[12] Root H. The association of diabetes and tuberculosis. N Engl J Med 1934;210:1-13.

[13] Restrepo BI, Fisher-Hoch SP, Crespo JG, et al. Type 2 diabetes and tuberculosis in a dynamic bi-national border population. Epidemiol Infect 2007;135(3):48391.

[14] Sosman M, Steidl J. Diabetic tuberculosis. Am J Roentgenol 1927;17:625.

[15] Weaver RA. Unusual radiographic presentation of pulmonary tuberculosis in diabetic patients. Am Rev Respir Dis 1974;109(1):162-3.

[16] Marais RM. Diabetes mellitus in black and coloured tuberculosis patients. S Afr Med J 1980;57(13):483-4.

[17] Al-Tawfiq JA, Saadeh BM. Radiographic manifestations of culture-positive pulmonary tuberculosis: cavitary or non-cavitary? Int J Tuberc Lung Dis 2009;13(3):36770.

[18] Segarra F, Sherman DS, Rodriguez-Aguero J. Lower lung field tuberculosis. Am Rev Respir Dis 1963;87(1):37-40.

[19] Hadlock FP, Park SK, Awe RJ, et al. Unusual radiographic findings in adult pulmonary tuberculosis. AJR Am J Roentgenol 1980;134(5):1015-8.

[20] Ikezoe J, Takeuchi N, Johkoh T, et al. CT appearance of pulmonary tuberculosis in diabetic and immunocompromised patients: comparison with patients who had no underlying disease. AJR Am J Roentgenol 1992;159(6):1175-9. 